\title{
Thermal entanglement in a triple quantum dot system
}

\author{
M. Urbaniak ${ }^{1}$, S.B. Tooski ${ }^{1,2}$, A. Ramšak ${ }^{2}$, and B.R. Bułka ${ }^{1, a}$ \\ 1 Institute of Molecular Physics, Polish Academy of Sciences, ul. M. Smoluchowskiego 17, 60-179 Poznań, Poland \\ 2 Faculty of Mathematics and Physics, University of Ljubljana and Jozef Stefan Institute, 1000 Ljubljana, Slovenia
}

Received 13 August 2013 / Received in final form 13 November 2013

Published online 11 December 2013

(c) The Author(s) 2013. This article is published with open access at Springerlink.com

\begin{abstract}
We present studies of thermal entanglement of a three-spin system in triangular symmetry. Spin correlations are described within an effective Heisenberg Hamiltonian, derived from the Hubbard Hamiltonian, with super-exchange couplings modulated by an effective electric field. Additionally a homogenous magnetic field is applied to completely break the degeneracy of the system. We show that entanglement is generated in the subspace of doublet states with different pairwise spin correlations for the ground and excited states. For the doublets with the same spin orientation one can observe nonmonotonic temperature dependence of entanglement due to competition between entanglement encoded in the ground state and the excited state. The mixing of the states with an opposite spin orientation or with quadruplets (unentangled states) always monotonically destroys entanglement. Pairwise entanglement is quantified using concurrence for which analytical formulae are derived in various thermal mixing scenarios. The electric field plays a specific role - it breaks the symmetry of the system and changes spin correlations. Rotating the electric field can create maximally entangled qubit pairs together with a separate spin (monogamy) that survives in a relatively wide temperature range providing robust pairwise entanglement generation at elevated temperatures.
\end{abstract}

\section{Introduction}

Entanglement is recognized to be a key resource in quantum information processing tasks such as quantum computation, teleportation and cryptography [1]. In recent years the two-qubit entanglement which includes variety of interactions have been studied extensively [2]. However, the three-qubit entanglement states have been revealed to hold advantage over the two-qubit states in quantum teleportation [3], dense coding [4] and quantum cloning [5]. DiVincenzo et al. [6] proposed quantum computations scheme to measure and control states by logical gates in a three-qubit spin system with exchange interactions in quantum dots, in which logical qubits are encoded in the doublet subspace (see also $[7,8]$ ). This scheme uses the advantage of the decoherence-free subspace (DFS) theory in which quantum information encoded over the entangled subspace of system states is robust against decoherence and error processes [9-13]. Recently experimental efforts have been undertaken to fabricate such triple quantum dot structures and to perform coherent spin manipulations [14-16].

Fast reliable spin manipulation in quantum dots is one of the most important challenges in spintronics and semiconductor-based quantum information. Recent experiments show that this goal can be better achieved in electrically gated quantum dot qubits $[17,18]$. There have been

\footnotetext{
${ }^{a}$ e-mail: bulka@ifmpan.poznan.pl
}

ongoing theoretical studies to manipulate spin properties of triple quantum dot systems [19-22] and to apply them in the quantum information precessing controlled by external electric field $[18,23]$. For the quantum computing most relevant is the ground-state entanglement and its relation with the spin-spin correlation functions and chirality. However, in realistic systems and for potential application to quantum information processing, it is crucial to understand also the entanglement stability at elevated temperatures, which is one of the main goals of this paper.

One of the significant goals for quantum computing and quantum communication is to find an entangled source in solid state systems at a finite temperature. The problem of entanglement between two qubits interacting via the Heisenberg interaction at a nonzero temperature was investigated by Nielsen [24]. A similar problem of the variation of entanglement with temperature and magnetic field in a one dimension finite Heisenberg chain were studied by Arnesen et al. [25], and they used for the first time the notion of thermal entanglement. Thereafter, the issue of entanglement in thermal equilibrium states has been the subject of a number of papers dealing with different aspects of the problem [26-30].

Recently experimental efforts have been undertaken to fabricate such triple quantum dot (TQD) structures [14-16] and to perform coherent spin manipulations according to the scheme proposed by DiVincenzo et al. [6]. It was shown that the quantum states of a coded qubit in a 


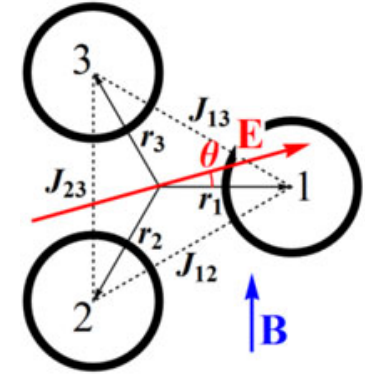

Fig. 1. Scheme of three quantum dots (qubits) in triangular symmetry under an effective electric filed $\mathbf{E}$ and a homogeneous magnetic field $\mathbf{B}$.

TQD indeed can be manipulated by tuning the gate voltages. Laird et al. [16] demonstrated experimentally the initialization, coherent exchange, and readout of a coded qubit based on TQD with three electrons by adopting a specific pulsing technique [17] that was first exploited to demonstrate coherent exchange and readout in a double dot system. Also TQD system with a triangular symmetry to observe spin frustration [31] was created and a state of single electron spin using single shot readout technique [32] was measured. Measuring single spins in many spins systems is the next goal that would allow to check results presented in this paper. To our knowledge, although several studies in the literature describe the entanglement in a three-qubit system [30], the thermal entanglement manipulated by an external electric field has not been considered so far. Here we present both analytical and numerical analysis of the thermal entanglement of triple quantum dots as a three-spin qubit described by an effective Heisenberg Hamiltonian at thermal equilibrium in the presence of both external magnetic and electric fields whose magnitude and direction provide additional degrees of freedom to control the entanglement. Since there is no unique measure of entanglement for tripartite entanglement [33,34], we have calculated monotone concurrence [35] as a measure of entanglement between two qubits of the three-qubit spin system. Then we study the combined influence of magnetic field strength and direction of electric field on entanglement of the system.

We also determine critical temperature $T_{0}$ for a chosen set of parameters of the system, beyond which concurrence vanishes in agreement with reference [36]. However, the concurrence can be a nonmonotonic function of temperature: at low temperatures regime the thermal mixing destroys the entanglement, while at higher temperatures one still can observe partial restoration of the entanglement. The studies add a new useful tool for manipulating the entanglement of the three-qubit Heisenberg model.

\section{Model of three spins in a quantum dot system}

We consider a model of three quantum dots (sites) in triangular geometry (Fig. 1) with three electrons, and the system is under influence of a homogeneous magnetic field. The system is described by the Hubbard Hamiltonian $[21,23]$ :

$$
\begin{aligned}
\hat{H}= & \sum_{i, \sigma} \tilde{\epsilon}_{i} n_{i \sigma}+t \sum_{i \neq j, \sigma}\left(c_{i \sigma}^{\dagger} c_{j \sigma}+\text { h.c. }\right)+U \sum_{i} n_{i \uparrow} n_{i \downarrow} \\
& -g \mu_{B} B_{z} \sum_{i, \sigma} \sigma n_{i \sigma} .
\end{aligned}
$$

We assume that at each quantum dot a single energy level $\epsilon_{i}$ is accessible for electrons. In an experiment the position of $\epsilon_{i}$ can be shifted in a fully controllable way by potentials applied to local gates. Since the symmetry of the system is essential in our considerations, it is more suitable to introduce an effective electric field $\mathbf{E}$ and express $\epsilon_{i} \equiv$ $\tilde{\epsilon_{0}}+e \mathbf{E} \cdot \mathbf{r}_{i}$ (see Fig. 1). Here, $e$ denotes the electron charge and $\mathbf{r}_{i}$ is the vector to the $i$-quantum dot. Later we will use $\tilde{\epsilon_{0}} \equiv \epsilon_{1}+\epsilon_{2}+\epsilon_{3}=0$ and express the polarization term as $\tilde{\epsilon}_{i} \equiv e \mathbf{E} \cdot \mathbf{r}_{i}=g_{E} \cos [\theta+(i-1) 2 \pi / 3], g_{E}=e E r$, $r_{i}=r-$ a length of $\mathbf{r}_{i}, \theta-$ an angle between the electric field $\mathbf{E}$ and $\mathbf{r}_{1}$. The second term in equation (1) describes the electron hopping between the nearest quantum dots. To make our considerations more transparent we assume that hopping is the same $t_{i j}=t$ for each pair $\{i j\}$. The third term is the onsite Coulomb interaction of electrons with opposite spins on the quantum dots. The last term is the spin interaction with an external magnetic field the Zeeman term. Here $\mu_{B}$ denotes the Bohr magneton, $g$ is the electron $g$-factor and the magnetic field $B_{z}$ is taken to be in the plane of the system - along the $z$-axis. (The magnetic field oriented perpendicular to the plane can lead to circulation of spin supercurrents which make the bipartite concurrence uniform [23]. This aspect will not be considered in the paper.)

If the onsite Coulomb interaction $U$ is much greater than the other parameters $t$ and $g_{E}$, we can operate in the space of singly occupied states transforming the Hubbard Hamiltonian to an effective Heisenberg Hamiltonian

$$
\hat{H}_{e f f}=\sum_{i<j} J_{i j}\left(\mathbf{S}_{\mathbf{i}} \cdot \mathbf{S}_{\mathbf{j}}-\frac{1}{4}\right)-h \sum_{i} S_{z_{i}},
$$

where $h=g \mu_{B} B_{z}$. The first term describes the superexchange coupling [37] (see also [38]) between spins with the parameter $J_{i j}$ calculated to the third order in $t / U[39]$ :

$$
J_{i j}=\frac{4 t^{2}}{U}+\frac{4 t^{2}\left(\tilde{\epsilon}_{j}-\tilde{\epsilon}_{i}\right)^{2}}{U^{3}}+\frac{8 t^{3}\left(2 \tilde{\epsilon}_{m}-\tilde{\epsilon}_{i}-\tilde{\epsilon}_{j}\right)}{U^{3}}
$$

where $i, j, m$ are 3 different indices of the quantum dots and $\tilde{\epsilon}_{i}$ is the local single electron level shifted by the electric field. (The parameters $J_{i j}$ can be derived also for a general case with different hoppings $t_{i j}$ - see [39].) The electric field is responsible for symmetry breaking in the system varying the superexchange parameters $J_{i j}$.

The states for three spins can be constructed from the two spin states, singlets and triplets, by adding an electron $[21,40]$. As a result one gets the quadruplet manifold and two doublet states with the total spin $S=3 / 2$ and $S=1 / 2$, respectively. The quadruplet states with 
$S_{z}=+3 / 2,+1 / 2$ are:

$$
\begin{aligned}
& \left|Q_{3 / 2}\right\rangle=|\uparrow \uparrow \uparrow\rangle, \\
& \left|Q_{1 / 2}\right\rangle=\frac{1}{\sqrt{3}}(|\downarrow \uparrow \uparrow\rangle+|\uparrow \downarrow \uparrow\rangle+|\uparrow \uparrow \downarrow\rangle),
\end{aligned}
$$

and similarly the states $\left|Q_{-3 / 2}\right\rangle,\left|Q_{-1 / 2}\right\rangle$ with the opposite spin direction. The quadruplet states with $S_{z}= \pm 1 / 2$ are the W states. The doublet states $\left({ }^{1 / 2} D_{S_{z}}\right)$ with $S_{z}= \pm 1 / 2$ can be constructed from the base states:

$$
\begin{aligned}
& \left|D_{1 / 2}\right\rangle_{1}=\frac{1}{\sqrt{2}}(|\uparrow \uparrow \downarrow\rangle-|\uparrow \downarrow \uparrow\rangle), \\
& \left|D_{1 / 2}\right\rangle_{2}=\frac{1}{\sqrt{6}}(2|\downarrow \uparrow \uparrow\rangle-|\uparrow \uparrow \downarrow\rangle-|\uparrow \downarrow \uparrow\rangle),
\end{aligned}
$$

and similarly the states $\left|D_{-1 / 2}\right\rangle_{1},\left|D_{-1 / 2}\right\rangle_{2}$ with the opposite spin direction. Notice that $\left|D_{S_{z}}\right\rangle_{1}$ is constructed from the singlet on the sites $\{23\}$ and adding an electron to the site 1 , while $\left|D_{S_{z}}\right\rangle_{2}$ is built from triplets on the sites $\{23\}$ and adding an electron to the site 1 . These base states constitute a doublet subspace used in DiVincenzo's scheme [6] for quantum computation based on three quantum semiconducting dot qubits (see also [8]). All four doublet eigenstates in the system can be expressed as:

$$
\left|D_{S_{z}}^{ \pm}\right\rangle=\cos \varphi^{ \pm}\left|D_{S_{z}}\right\rangle_{1}+\sin \varphi^{ \pm}\left|D_{S_{z}}\right\rangle_{2}
$$

The phase $\varphi^{ \pm}$is given by the relation:

$$
\cot \varphi^{ \pm}=\frac{J_{12}-2 J_{23}+J_{31} \pm 2 \Delta}{\sqrt{3}\left(J_{31}-J_{12}\right)},
$$

where $\Delta$ is given by equation (13). The symmetry of $\varphi^{ \pm}$ depends on the choice of the base of doublet states equations (6) and (7).

The existence of electric and magnetic field breaks the fourfold degeneracy of quadruplet and doublet states in the symmetric system. Solving the eigenvalue problem for the effective Hamiltonian equation (2) one finds the energies:

$$
\begin{aligned}
& E_{Q_{S_{z}}}=-h S_{z} \\
& E_{D_{S_{z}}^{ \pm}}=-\frac{3}{2} J \pm \frac{1}{2} \Delta-h S_{z},
\end{aligned}
$$

for quadruplet and doublets, respectively. Here,

$$
\begin{aligned}
& J=\left(J_{12}+J_{23}+J_{31}\right) / 3 \\
& \Delta=\sqrt{J_{12}^{2}+J_{23}^{2}+J_{31}^{2}-J_{12} J_{31}-J_{12} J_{23}-J_{31} J_{23}} .
\end{aligned}
$$

$\Delta$ is responsible for splitting of eigenstates due to the presence of electric field. Therefore we call this effect as the spin Stark effect [23]. A scheme of energy levels split by the magnetic field $h$ is shown in Figure 2.

Experimentally it is possible to obtain similar system of three quantum dots [41] but in reality it will never be perfectly symmetrical resulting in different hopping integrals $t_{i j}$ and different $U_{i}$ on each dot. However

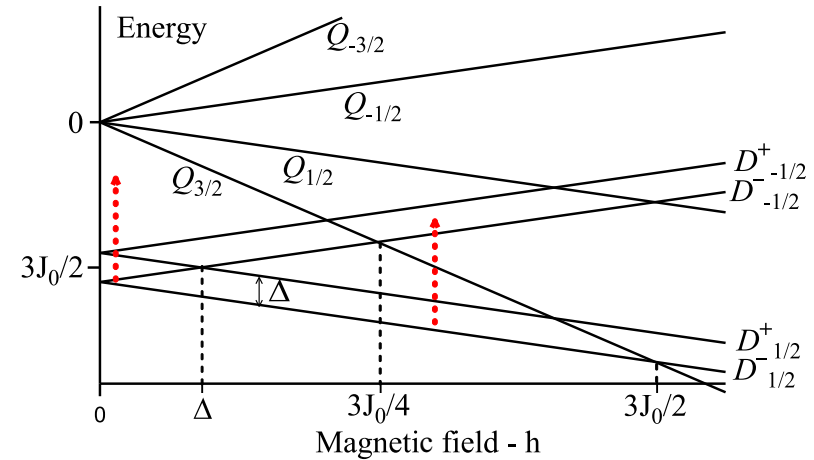

Fig. 2. Scheme of energy levels with the Zeeman splitting of the doublet $\left(D_{ \pm 1 / 2}^{ \pm}\right)$and the quadruplet $\left(Q_{ \pm 3 / 2, \pm 1 / 2}\right)$ states. Red dashed arrows show two considered situations of thermal mixing of states with raising temperature in the system. The first one is for weak magnetic field $h \ll \Delta$ and the second is for strong magnetic field $\left(\Delta \ll h<3 J_{0} / 2\right)$. Here $J_{0} \equiv 4 t^{2} / U$ and we assume $\Delta \ll J_{0}$.

if the canonical transformation is used for the general case [39] one can nevertheless get any desired value of $J_{i j}$ by modifying local dot potentials $\tilde{\epsilon}_{i}$.

Notice that there are no processes for transition between the subspaces with the total spin $S=1 / 2$ and $S=3 / 2$ for our model (2). The doublet subspace is the decoherence free subspace (DFS) $[12,13]$ and entanglement encoded in this subspace is robust against decoherence with electrodes (an external bath system). This is a main advantage of the quantum computation scheme proposed by DiVincenzo et al. [6].

\section{Spin correlations and entanglement}

We assume that the considered spin system is in thermal equilibrium with the environment and is described by the density matrix [42]:

$$
\rho=\sum_{\alpha=1}^{8} z_{\alpha}\left|\psi_{\alpha}\right\rangle\left\langle\psi_{\alpha}\right|
$$

where $z_{\alpha}=e^{-\beta E_{\alpha}} / Z$ are the Boltzmann factors with $Z=\sum_{\alpha} z_{\alpha}, \beta=1 / k_{B} T, E_{\alpha}$ are eigenenergies and the corresponding eigenvectors are $\left|\psi_{\alpha}\right\rangle \in\left\{\left|D_{1 / 2}^{-}\right\rangle,\left|D_{-1 / 2}^{-}\right\rangle\right.$, $\left.\left|D_{1 / 2}^{+}\right\rangle,\left|D_{-1 / 2}^{+}\right\rangle,\left|Q_{3 / 2}\right\rangle,\left|Q_{1 / 2}\right\rangle,\left|Q_{-1 / 2}\right\rangle,\left|Q_{-3 / 2}\right\rangle\right\}$. We would like to study entanglement between spins on two sites $\{i j\}$ in the considered three qubit system. To this end we use the reduced density matrix $\rho_{i j}=\operatorname{Tr}_{m \neq i, j}[\rho]$, which in the standard basis $\{\uparrow \uparrow, \uparrow \downarrow, \downarrow \uparrow, \downarrow \downarrow\}$ is expressed as:

$$
\rho_{i j}=\left(\begin{array}{cccc}
\mu_{i j} & 0 & 0 & 0 \\
0 & \xi_{i j} & \gamma_{i j} & 0 \\
0 & \gamma_{i j} & \nu_{i j} & 0 \\
0 & 0 & 0 & \delta_{i j}
\end{array}\right) .
$$


Explicitly for the effective Heisenberg Hamiltonian equation (2) the elements $\gamma_{i j}, \mu_{i j}$ and $\delta_{i j}$ are expressed as:

$$
\begin{aligned}
& \mu_{i j}=\frac{1}{3}\left[\left(z_{D_{1 / 2}^{+}}-z_{D_{1 / 2}^{-}}\right) \cos \left(\tilde{\theta}_{i j}\right)+z_{D_{1 / 2}}\right]+z_{Q_{3 / 2}}+\frac{z_{Q_{1 / 2}}}{3}, \\
& \gamma_{i j}=\left[\left(z_{D^{+}}-z_{D^{-}}\right) 2 \cos \left(\tilde{\theta}_{i j}\right)-z_{D}\right]+\frac{z_{Q_{1 / 2}}+z_{Q_{-1 / 2}}}{3}, \\
& \delta_{i j}=\frac{1}{3}\left[\left(z_{D_{-1 / 2}^{+}}-z_{D_{-1 / 2}^{-}}\right) \cos \left(\tilde{\theta}_{i j}\right)+z_{D_{-1 / 2}}\right] \\
& +z_{Q_{-3 / 2}}+\frac{z_{Q_{-1 / 2}}}{3} \text {, }
\end{aligned}
$$

where

$$
\begin{aligned}
z_{D} & \equiv z_{D_{-1 / 2}^{-}}+z_{D_{-1 / 2}^{+}}+z_{D_{+1 / 2}^{-}}+z_{D_{+1 / 2}^{+}}, \\
z_{D^{ \pm}} & \equiv z_{D_{-1 / 2}^{ \pm}}+z_{D_{+1 / 2}^{ \pm}}, \\
z_{D_{ \pm 1 / 2}} & \equiv z_{D_{ \pm 1 / 2}^{+}}+z_{D_{ \pm 1 / 2}^{-}} .
\end{aligned}
$$

In equation (16) $\tilde{\theta}_{i j}=2 \varphi^{-}+q_{i j}, \varphi^{-}$is the phase given by equation (9) and $q_{i j}=\{0,-2 \pi / 3,2 \pi / 3\}$ for site pairs $\{i j\}=\{23,13,12\}$, respectively. The form of $q_{i j}$ comes from the chosen symmetry of the base states equations (6) and (7). In derivation of equation (16) the relation: $\cos \left(2 \varphi^{-}+q_{i j}\right)=-\cos \left(2 \varphi^{+}+q_{i j}\right)$ was used which can be proved using equations (9) and (13).

We use concurrence $C_{i j}$ as a measure of pair-wise entanglement [35], which is given by $C_{i j}=\max \left\{\Lambda_{i j}=\right.$ $\left.\lambda_{\max }-\sum_{i} \lambda_{i}, 0\right\}$, where $\lambda_{i}$ are eigenvalues of the matrix $\rho_{i j} \cdot \tilde{\rho}_{i j}$ and $\tilde{\rho}_{i j}=\left(\sigma_{y} \otimes \sigma_{y}\right) \cdot \rho_{i j}^{*} \cdot\left(\sigma_{y} \otimes \sigma_{y}\right)$ is spin flipped matrix. For spin rings and the density matrix of the form equation (15) concurrence can be expressed as [43]:

$$
C_{i j}=2 \max \left\{\left|\gamma_{i j}\right|-\sqrt{\mu_{i j} \delta_{i j}}, 0\right\} .
$$

In further investigations of thermal entanglement we use the parameters equation (16) for the effective Heisenberg model equation (2) with superexchange couplings. The exact solutions of thermal concurrence together with the analysis of several special cases and relationships between spin correlation function and concurrence will be presented below. It is worth to notice that these results are valid for general case of any Heisenberg model, in which one can change symmetry of the spin system by a modulation of exchange couplings $J_{i j}$.

\subsection{Ground state}

First we present studies for entanglement in the ground state, which will be a reference system for further studies of thermal entanglement when temperature leads to mixing with excited states. For magnetic field $0<h<$ $3 J_{0} / 2$ the ground state is $\left|D_{1 / 2}^{-}\right\rangle=\cos \varphi^{-}\left|D_{1 / 2}\right\rangle_{1}+$ $\sin \varphi^{-}\left|D_{1 / 2}\right\rangle_{2}$ (see Fig. 2). The symmetry of the system is controlled by orientation of the electric field, by its angle $\theta$. Using equations (18) and (16) one can easily show that $C_{i j}$ reads:

$$
C_{i j}=\frac{1}{3}\left|2 \cos \left(\tilde{\theta}_{i j}\right)+1\right| .
$$

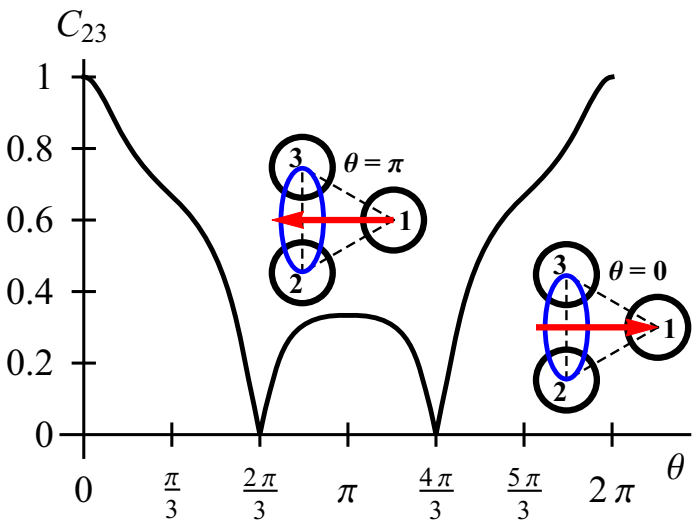

Fig. 3. Plot of concurrence $C_{23}$ for the doublet ground state $\left|D_{1 / 2}^{-}\right\rangle$as a function of angle $\theta$ of electric field. The insets show the orientation of electric field (red arrow) in relation to the considered pair for $\theta=0$ and $\theta=\pi$, when concurrence becomes maximal $C_{23}=1$ and $C_{23}=1 / 3$, respectively. Parameters used for computation: $U=20, g_{E}=1, t=1$.

Comparing with a spin-spin correlation function:

$$
\left\langle\mathbf{S}_{i} \cdot \mathbf{S}_{j}\right\rangle=\frac{1}{4}\left[-2 \cos \left(\tilde{\theta}_{i j}\right)-1\right]
$$

one finds the following relation [23]:

$$
C_{i j}=\frac{4}{3}\left|\left\langle\mathbf{S}_{i} \cdot \mathbf{S}_{j}\right\rangle\right| \text {. }
$$

As a result it appears that in this case, the concurrence and the expectation value for the spin correlation function contain the same quantum information.

The plot of concurrence $C_{23}$ equation (19) as a function of $\theta$ is shown in Figure 3. Here we choose the pair $\{23\}$ because it is most convenient for the doublet basis equations (6)-(7). Due to the symmetry one can obtain identical plots for $C_{12}$ and $C_{13}$ shifting the plot for $C_{23}$ by $\theta= \pm 2 \pi / 3$. The parameters for numerical computations were taken: Coulomb repulsion $U=20$, hopping integral $t=1$ and electric field strength $g_{E}=1$, which ensure the requirement of $U \gg t, g_{E}$ for the canonical transformation to Heisenberg Hamiltonian. For this strength of the electric field the parameter $\tilde{\theta}_{23} \approx \theta$. One can see that the concurrence reaches maximum $C_{23}=1$ when the electric field is perpendicular to the considered pair and oriented towards the site 1 . Then the local electron energy is minimal and the coupling $J_{23}$ is maximal, $J_{23}>J_{12}=J_{13}$. In this case the ground state of the system becomes $\left|D_{1 / 2}\right\rangle_{1}$, equation (6), which describes singlet on the pair $\{23\}$ and separated spin at the site 1 . This means monogamy, because the concurrence between the other sites, $C_{12}=C_{13}=0$ [44]. Two minima $C_{23}=0$ at $\theta=2 \pi / 3$ and $4 \pi / 3$ correspond to singlets on pairs $\{12\}$ and $\{13\}$, respectively. Additionally one can see local maximum of $C_{i j}=1 / 3$ at $\theta=\pi$ corresponding to the situation when the local electron energy is maximal and the coupling $J_{23}$ is minimal, $J_{23}<J_{12}=J_{13}$, with the ground state $\left|D_{1 / 2}\right\rangle_{2}$, equation (7), and the correlation between 
the spins is ferromagnetic, $\left\langle\mathbf{S}_{2} \cdot \mathbf{S}_{3}\right\rangle=+1 / 4$. Notice that switching the orientation of electric field from $\theta=0$ to $\theta=\pi$ causes a rotation of DiVincenzo's qubit from the state $\left|D_{1 / 2}\right\rangle_{1}$ to $\left|D_{1 / 2}\right\rangle_{2}$. In the following we will investigate how these features survive thermal mixing with excited states.

\subsection{Mixed states}

For finite temperature $T>0$ we assume that the system is in thermal equilibrium and the states are populated according the Boltzmann factor $z_{\alpha}$. Note that for a non-degenerate ground state the thermal entanglement at $T=0$ is identical to the entanglement of the ground state considered in previous subsection. The arrangement of eigenenergies $E_{\alpha}$ in this case mainly depends on the magnetic field $h$ (see Fig. 2). On the other hand the doublet states $\left|D_{ \pm 1 / 2}^{ \pm}\right\rangle$, equation (8), depend on the angle of electric field $\theta$ which modulates parameters $\varphi^{ \pm}$and corresponding $\tilde{\theta}_{i j}$. We would like to show how the concurrence $C_{i j}(\theta)$ for the ground state equation (19) changes with an increase of temperature $T$, resulting in mixing with excited states, for different magnetic fields $h$. In particular, two cases of weak and strong magnetic field will be considered, as they correspond to different order of eigenstates that qualitatively change system thermal properties and entanglement.

Using equations (18) and (16) we derive a general formula for the concurrence taking into account mixing with all states:

$$
\begin{aligned}
C_{i j}= & \frac{1}{3}\left[\mid z_{D}-2\left(z_{Q_{-1 / 2}}+z_{Q_{+1 / 2}}\right)-2\left(z_{D^{+}}-z_{D^{-}}\right)\right. \\
& \left.\times \cos \left(\tilde{\theta}_{i j}\right) \mid-2 \sqrt{K_{i j}^{\downarrow} K_{i j}^{\uparrow}}\right],
\end{aligned}
$$

where

$$
\begin{aligned}
K_{i j}^{\uparrow}= & z_{D_{+1 / 2}}+z_{Q_{+1 / 2}}+3 z_{Q_{+3 / 2}} \\
& -\left(z_{D_{+1 / 2}^{-}}-z_{D_{+1 / 2}^{+}}\right) \cos \left(\tilde{\theta}_{i j}\right)
\end{aligned}
$$

and similarly $K_{i j}^{\downarrow}$ with opposite spin direction. Here the Boltzmann coefficients are described by equation (17). Because the electric field acts only on doublets states, the concurrence equation (22) becomes independent on $\theta$ when the doublets $\left|D_{S_{z}}^{-}\right\rangle$and $\left|D_{S_{z}}^{+}\right\rangle$are thermally mixed, i.e., for $z_{D^{-}} \approx z_{D^{+}}$.

Dependence of the concurrence on magnetic field is presented on the $h-T$ plots in Figure 4 for the case $\theta=0$ and $\pi$, which correspond to the electric field $\mathbf{E}$ perpendicular to the bond $\{23\}$ and directed from and to the site 1 , respectively. In the absence of the magnetic field $(h=0)$ the states with opposite spin orientations are degenerated and equally populated. In the ground state the concurrence is given by $C_{i j}(T=0, h=0)=\max \left\{\cos \left(\tilde{\theta}_{i j}\right), 0\right\}$. Switching on the magnetic field removes degeneracy and results in a large increase of concurrence up to $h<\Delta$. For higher

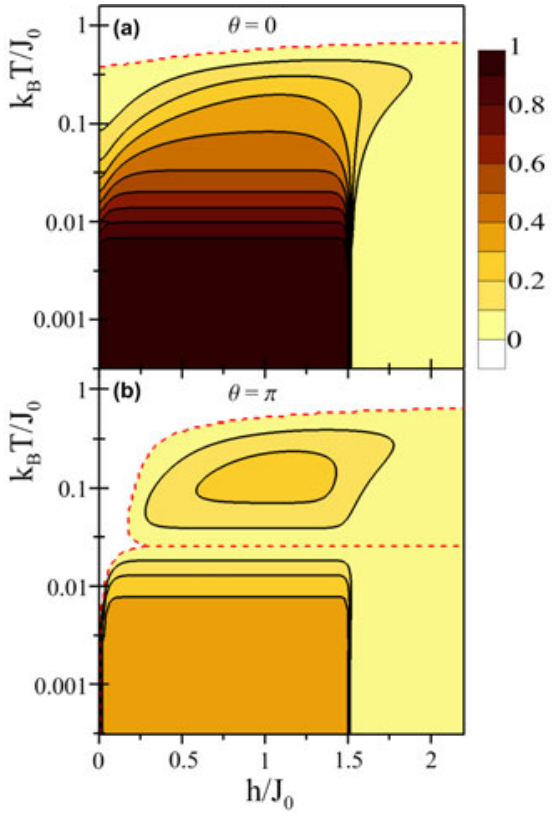

Fig. 4. Contour plot for concurrence $C_{23}$ in the $h-T$ plane for an angle (a) $\theta=0$ and (b) $\theta=\pi$ of electric field. The dashed (red) curve corresponds to $T_{0}$ when $C_{23}=0$ and the white area shows the unentangled region with $C_{12}=0$. Parameters used for computation: $U=20, g_{E}=1, t=1$, which give $J_{0} \equiv 4 t^{2} / U=0.2, \Delta \approx 0.006=0.03 J_{0}$.

magnetic fields the doublets $\left|D_{1 / 2}^{-}\right\rangle$and $\left|D_{1 / 2}^{+}\right\rangle$with the same spin orientations play a crucial role in low temperatures. Concurrence $C_{i j}(T)$ increases with $h$, because the larger Zeeman splitting makes weaker thermal mixing. For a very large magnetic field, $h>3 J_{0} / 2$, the unentangled quadruplet $Q_{3 / 2}$ is the ground state and hence the concurrence diminishes for very strong magnetic fields $[25,45]$. In this range the concurrence is exponentially small due to thermal excitations to the doublets.

An interesting result is presented in Figure $4 \mathrm{~b}$ where the concurrence shows a nonmonotonic temperature dependence, first at low temperatures it decreases to zero and one observes restoration of entanglement at high temperatures. We show below that this is an effect of a specific interplay between entanglement of the ground state $\left|D_{1 / 2}^{-}\right\rangle$ and the excited state $\left|D_{1 / 2}^{+}\right\rangle$.

Figure 5 shows contour plots of $C_{23}$ in the plane of $\theta$ and $T$ for two different values of magnetic field $h$, weak $(h<\Delta)$ and strong $\left(\Delta \ll h<3 J_{0} / 2\right)$. One can see two local maxima $C_{23}=1$ and $C_{23}=1 / 3$ for the angles $\theta=0$ and $\theta=\pi$, as well as two minima with $C_{23}=0$ at $\theta=2 \pi / 3$ and $4 \pi / 3$. For weak magnetic field $C_{23}(T)$ monotonously decreases with an increase of $T$, while for high magnetic field $C_{23}(T)$ is a nonmonotonic function at $\theta=\pi$ but it is still monotonic at $\theta=0$. Let us analyze thermal entanglement in these two cases in details.

Weak magnetic field. At low temperatures one can consider mixing of two states only: the ground state $\left|D_{1 / 2}^{-}\right\rangle$. These states have the same symmetry (with the same phase $\varphi^{-}$) but opposite spin orientation. In this case 


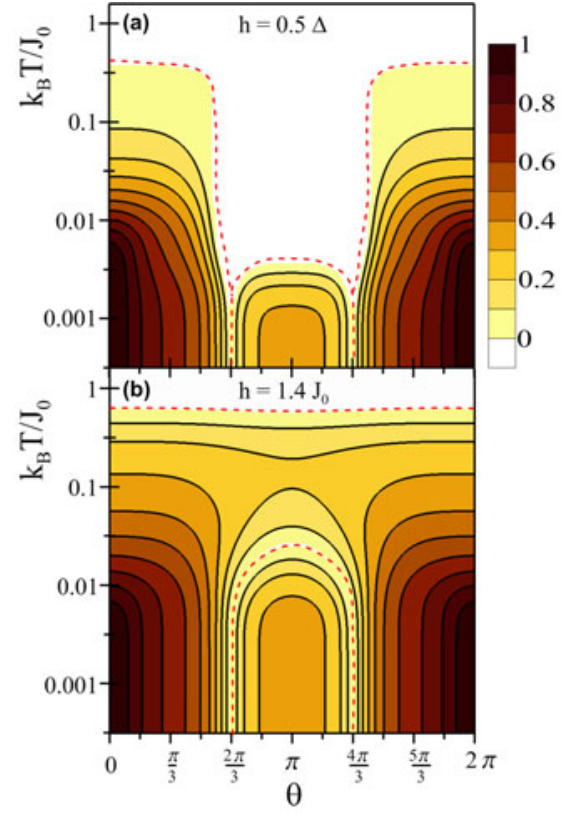

Fig. 5. Contour plot for concurrence $C_{23}$ in the $\theta-T$ plane (a) for a weak $\left[h=0.5 \Delta \approx 0.015 J_{0}\right]$, and $(\mathrm{b})$ strong $\left(h=1.4 J_{0}\right)$ magnetic field. The two magnetic field regimes correspond to dashed arrows on energy plot 2. Parameters are the same as in Figure 4.

one can take into account only the Boltzmann coefficients $z_{D_{1 / 2}^{-}}$and $z_{D_{-1 / 2}^{-}}$in equation (22) and write the concurrence as:

$$
\begin{aligned}
C_{i j}= & \max \left\{\frac{1}{3}\left|2 \cos \left(\tilde{\theta}_{i j}\right)+1\right|\right. \\
& \left.-\frac{2}{3} \sqrt{z_{D_{1 / 2}^{-}} z_{D_{-1 / 2}^{-}}}\left[1-\cos \left(\tilde{\theta}_{i j}\right)\right], 0\right\} .
\end{aligned}
$$

Comparing this result with the concurrence equation (19) for the ground state one can see that the second term in equation (24) describes reduction of the concurrence due to thermal mixing of the states. Notice that for $\cos \left(\tilde{\theta}_{i j}\right)=1$ the mixing term vanishes and $C_{i j}=1$ is sustained. It means that thermal entanglement is resistant to temperature rise till the higher energy states $\left(\left|D_{ \pm 1 / 2}^{+}\right\rangle\right)$ become populated.

The reduction term is proportional to $\left[z_{D_{1 / 2}^{-}} z_{D_{-1 / 2}^{-}}\right]^{1 / 2} \approx e^{-\beta h / 2}$. This result may be compared to the one obtained by Gunlycke et al. [46] for the Ising model in a perpendicular magnetic field, where the thermal mixture of two pure qubit states cause the reduction of concurrence proportional to $e^{-\beta h}$. Such a result is valid for any thermal mixing of pure states $\left|\psi_{n}\right\rangle$ with no spin-flip overlap, i.e. for $\left\langle\psi_{n}\left|\sigma_{y} \otimes \sigma_{y}\right| \psi_{m}\right\rangle=0$. In our case, however, spin-flip overlap of mixed stated does exist and the reduction of entanglement is lower, as $e^{-\beta h / 2}$.

At high temperatures $k_{B} T \gg h$ all states are populated and one may assume total mixing between the states with the opposite spins i.e.: $z_{D_{1 / 2}^{-}}=z_{D_{-1 / 2}^{-}}=z_{-}, z_{D_{1 / 2}^{+}}=$ $z_{D_{-1 / 2}^{+}}=z_{+}$as well as $z_{Q_{ \pm 3 / 2}}=z_{Q_{ \pm 1 / 2}}=z_{Q}$. Then a mixed state of the system can be written in the form: $\rho=z_{-} \rho^{-}+z_{+} \rho^{+}+z_{Q} \rho^{Q}$, where $\rho^{ \pm}=\sum_{S_{z}}\left|D_{S_{z}}^{ \pm}\right\rangle\left\langle D_{S_{z}}^{ \pm}\right|$ and $\rho^{Q}=\sum_{S_{z}}\left|Q_{S_{z}}\right\rangle\left\langle Q_{S_{Z}}\right|$. Partial concurrences of those constituent states: $C_{i j}\left(\rho^{ \pm, Q}\right)=\max \left\{\Lambda_{i j}^{ \pm, Q}, 0\right\}$ can be used to express concurrence $C_{i j}(\rho)$ :

$$
C_{i j}=\max \left\{z_{+} \Lambda_{i j}^{+}+z_{-} \Lambda_{i j}^{-}+3 z_{Q} \Lambda_{i j}^{Q}, 0\right\}
$$

where $\Lambda_{i j}^{ \pm, Q}=2\left(\left|\gamma_{i j}\right|-\left|\mu_{i j}\right|\right)$, equation (18). It shows that concurrence is expressed as thermal redistribution with additional factor 3 for quadruplet states. Using equation (16) we get:

$$
C_{i j}=\max \left\{\left(z_{-}-z_{+}\right) \cos \left(\tilde{\theta}_{i j}\right)-z_{Q}, 0\right\} .
$$

Strong magnetic field. For the case of strong magnetic field $\left(\Delta<h<3 J_{0} / 2\right)$ we have at low temperatures a mixture of two lowest states $D_{1 / 2}^{-}$and $D_{1 / 2}^{+}$with the same spin orientation. The symmetry of these states is different and characterized by different phases $\varphi^{-}$and $\varphi^{+}$. In this limit the concurrence equation (22) can be expressed as:

$$
C_{i j}=\frac{1}{3}\left|\left(z_{D_{1 / 2}^{-}}-z_{D_{1 / 2}^{+}}\right) 2 \cos \left(\tilde{\theta}_{i j}\right)+\left(z_{D_{1 / 2}^{-}}+z_{D_{1 / 2}^{+}}\right)\right| .
$$

Here the dependence on $\tilde{\theta}_{i j}$ exhibits specific interplay between entanglement in the doublet states. For $\cos \left(\tilde{\theta}_{i j}\right)>0$ (i.e. for $\theta \in[0,2 \pi / 3]$ and $\theta \in[4 \pi / 3,2 \pi]$ in the case presented in Fig. 5) the concurrence decreases monotonously with increasing $T$. On the other hand, if $\cos \left(\tilde{\theta}_{i j}\right)<0$ (for $\theta \in[2 \pi / 3,4 \pi / 3]$ in Fig. 5), the entanglement dominated by the excited state is larger than the one of the ground state. In this range the expectation value of the spin correlation function $\left\langle\mathbf{S}_{i} \cdot \mathbf{S}_{j}\right\rangle_{D_{1 / 2}^{-}}>0$ for the state $\left|D_{1 / 2}^{-}\right\rangle$, whereas the expectation value $\left\langle\mathbf{S}_{i} \cdot \mathbf{S}_{j}\right\rangle_{D_{1 / 2}^{+}}<0$ for the state $\left|D_{1 / 2}^{+}\right\rangle$. The specific interplay between entanglement of both states leads to a reduction of $C_{i j}$ to zero for small $T$, and its reconstruction for higher $T$ as entanglement coming from the excited state becomes dominant. One can calculate the critical temperature $k_{B} T_{0}=\Delta / \ln 3$ at which $C_{i j}=0$ due to perfect compensation of the both contributions from the state $D_{1 / 2}^{-}$and $D_{1 / 2}^{+}$.

Similar situation when entanglement is restored one can observe for very strong magnetic field $h>3 J_{0} / 2$ with quadruplet $\left|Q_{3 / 2}\right\rangle$ as the ground state $[25,45]$. Quadruplet is unentangled state but for higher $T$ doublets become populated which results restoration of concurrence.

Spin-spin correlation functions. One can also find the formula for spin-spin correlation functions for the mixed state equation (18) which reads:

$$
\left\langle\mathbf{S}_{i} \cdot \mathbf{S}_{j}\right\rangle=\frac{1}{4}\left(-z_{D}+z_{Q}+2\left(z_{D^{+}}-z_{D^{-}}\right) \cos \left(\tilde{\theta}_{i j}\right)\right) .
$$


Comparing general formula for concurrence equation (22) and spin-spin correlation functions equation (28) one can see that it is not possible to simply relate them in a general case. However, at small temperature $T$ and magnetic field $\Delta<h<3 J_{0} / 2$ only doublets $\left|D_{+1 / 2}^{ \pm}\right\rangle$with spin up are populated (see the energy plot in Fig. 2) and the relation between $C_{i j}$ and $\left\langle\mathbf{S}_{i} \cdot \mathbf{S}_{j}\right\rangle$ can be expressed as: $C_{i j}=$ $\frac{4}{3}\left|\left\langle\mathbf{S}_{i} \cdot \mathbf{S}_{j}\right\rangle\right|$ as for the case of ground state equation (21). On the other hand, in the case of very small $h \ll T$ when the Zeeman spitting is negligible and the states with opposite spin direction are equally populated, the relation changes to $C_{i j}=\max \left\{-2\left\langle\mathbf{S}_{i} \cdot \mathbf{S}_{j}\right\rangle-\frac{1}{2}, 0\right\}$ [26]. In the general case the relation between $C_{i j}$ and $\left\langle\mathbf{S}_{i} \cdot \mathbf{S}_{j}\right\rangle$ lies somewhere between those two extrema

$$
\max \left\{-2\left\langle\mathbf{S}_{i} \cdot \mathbf{S}_{j}\right\rangle-\frac{1}{2}, 0\right\} \leq C_{i j} \leq \frac{4}{3}\left|\left\langle\mathbf{S}_{i} \cdot \mathbf{S}_{j}\right\rangle\right| .
$$

\section{Summary}

Summarizing, we have investigated thermal pairwise entanglement in a triangular system of three coherently coupled semiconducting quantum dots. Spin correlations have been described within the effective Heisenberg Hamiltonian in which exchange coupling constants are derived from the Hubbard model using the canonical perturbation theory and tracing out double occupied states. The investigations included the Zeeman splitting caused by the magnetic field as well as symmetry breaking by the electric field (the spin Stark effect). We have shown that spin entanglement is generated by the doublet states. Rotating the electric field one can change the entanglement, creating maximum entanglement in a chosen pair of qubits together with a separate uncoupled spin (a spin dark state). For a specific symmetry one can set the system in the ground state either in state $\left|D_{1 / 2}\right\rangle_{1}$ (for $\theta=0$ ) or in state $\left|D_{1 / 2}\right\rangle_{2}$ (for $\theta=\pi$ ) and by manipulating the orientation $\theta$ of the electric field one can easily prepare the qubit in a desired state on the Bloch sphere. Our studies of entanglement indicate that the poles on the Bloch sphere (corresponding for $\left|D_{1 / 2}\right\rangle_{1}$ and $\left.\left|D_{1 / 2}\right\rangle_{2}\right)$ are the most stable and easiest to prepare (see Fig. 3).

In small clusters of coupled electrons with strong Coulomb repulsion it is not uncommon to find states corresponding to perfectly entangled qubit pairs. However, in general such states can be unstable with respect to charge fluctuations, coupling to energetically near states excited due to elevated temperature, small external magnetic fields or due to the coupling to external charge reservoirs. In this paper we concentrated on the properties of charge-transfer isolated TQD, but in contact with thermal bath in the presence of an external magnetic field, while the analysis of the influence of the charge-transfer coupling to external leads will be presented elsewhere.

As expected, at finite temperatures the entanglement will in general be reduced due to thermal mixing with excited states. In particular, our studies show that the state $\left|D_{1 / 2}\right\rangle_{1}$ is robust on thermal mixing and stable for relatively large temperatures (see Figs. 4 and 5). In contrast the state $\left|D_{1 / 2}\right\rangle_{2}$ is less stable on temperature. For a high temperature the excited states (with different pairwise correlations between spins) will come into the play. Concurrence depends on relative thermal occupation of both states and it can be a nonmonotonic function of temperature. At the regime of very low temperatures and not too large magnetic field (the ground state is doublet), thermal mixing reduces the concurrence. However, at higher temperatures, the entanglement can be restored due to spin correlations in the excited state. As expected, thermal mixing between the doublets with opposite spin orientation, $S_{z}=+1 / 2$ and $S_{z}=-1 / 2$, reduces the entanglement. Quadruplets (the unentangled states) lead to total destruction of entanglement.

This work was supported by the National Science Centre under the contract DEC-2012/05/B/ST3 /03208 and by the EU project Marie Curie ITN NanoCTM. A.R. would like to acknowledge also support from ARRS under contract No. P1-0044.

\section{References}

1. M. Nielsen, I. Chuang, Quantum Computation and Quantum Information, Cambridge Series on Information and the Natural Sciences (Cambridge University Press, 2000)

2. R. Horodecki, P. Horodecki, M. Horodecki, K. Horodecki, Rev. Mod. Phys. 81, 865 (2009)

3. A. Karlsson, M. Bourennane, Phys. Rev. A 58, 4394 (1998)

4. J.C. Hao, C.F. Li, G.C. Guo, Phys. Rev. A 63, 054301 (2001)

5. D. Bruß, D.P. DiVincenzo, A. Ekert, C.A. Fuchs, C. Macchiavello, J.A. Smolin, Phys. Rev. A 57, 2368 (1998)

6. D.P. DiVincenzo, D. Bacon, J. Kempe, G. Burkard, K.B. Whaley, Nature 408, 339 (2000)

7. Y.S. Weinstein, C.S. Hellberg, Phys. Rev. A 72, 022319 (2005)

8. D. Bacon, Ph.D. thesis, University of California, Berkeley, 2001, arXiv:quant-ph/0305025

9. P. Zanardi, M. Rasetti, Phys. Rev. Lett. 79, 3306 (1997)

10. P. Zanardi, F. Rossi, Phys. Rev. Lett. 81, 4752 (1998)

11. B.H. Fong, S.M. Wandzura, Quantum Info. Comput. 11, 1003 (2011)

12. D.A. Lidar, I.L. Chuang, K.B. Whaley, Phys. Rev. Lett. 81, 2594 (1998)

13. J. Kempe, D. Bacon, D.A. Lidar, K.B. Whaley, Phys. Rev. A 63, 042307 (2001)

14. T. Takakura, M. Pioro-Ladriere, T. Obata, Y.S. Shin, R. Brunner, K. Yoshida, T. Taniyama, S. Tarucha, Appl. Phys. Lett. 97, 212104 (2010)

15. L. Gaudreau, G. Granger, A. Kam, G.C. Aers, S.A. Studenikin, P. Zawadzki, M. Pioro-Ladriere, Z.R. Wasilewski, A.S. Sachrajda, Nat. Phys. 8, 54 (2012)

16. E.A. Laird, J.M. Taylor, D.P. DiVincenzo, C.M. Marcus, M.P. Hanson, A.C. Gossard, Phys. Rev. B 82, 075403 (2010)

17. J.R. Petta, A.C. Johnson, J.M. Taylor, E.A. Laird, A. Yacoby, M.D. Lukin, C.M. Marcus, M.P. Hanson, A.C. Gossard, Science 309, 2180 (2005) 
18. Z. Shi, C.B. Simmons, J.R. Prance, J.K. Gamble, T.S. Koh, Y.P. Shim, X. Hu, D.E. Savage, M.G. Lagally, M.A. Eriksson, M. Friesen, S.N. Coppersmith, Phys. Rev. Lett. 108, 140503 (2012)

19. Y. Li, C. Yannouleas, U. Landman, Phys. Rev. B 76, 245310 (2007)

20. C.Y. Hsieh, P. Hawrylak, Phys. Rev. B 82, 205311 (2010)

21. B.R. Bułka, T. Kostyrko, J. Łuczak, Phys. Rev. B 83, 035301 (2011)

22. C.Y. Hsieh, Y.P. Shim, M. Korkusinski, P. Hawrylak, Rep. Prog. Phys. 75, 114501 (2012)

23. J. Łuczak, B.R. Bułka, J. Phys.: Condens. Matter 24, 375303 (2012)

24. M.A. Nielsen, Ph.D. Dissertation, The University of New Mexico, 1998, arXiv:quant-ph/0011036

25. M.C. Arnesen, S. Bose, V. Vedral, Phys. Rev. Lett. 87, $017901(2001)$

26. A. Ramsak, J. Mravlje, T. Rejec, A. Lautar, Europhys. Lett. 86, 40003 (2009)

27. S. El Shawish, A. Ramšak, J. Bonča, Phys. Rev. B 75, $205442(2007)$

28. A. Ramsak, I. Sega, J.H. Jefferson, Phys. Rev. A 74, 010304 (2006)

29. G.F. Zhang, Phys. Rev. A 75, 034304 (2007)

30. X.L. Huang, J.L. Guo, X.X. Yi, Phys. Rev. A 80, 054301 (2009)

31. S. Andergassen, Nature 495, 321 (2013)

32. A. Morello, J.J. Pla, F.A. Zwanenburg, K.W. Chan, K.Y. Tan, H. Huebl, M. Mottonen, C.D. Nugroho, C. Yang, J.A. van Donkelaar, A.D.C. Alves, D.N. Jamieson, C.C. Escott, L.C.L. Hollenberg, R.G. Clark, A.S. Dzurak, Nature 467, $687(2010)$

33. W. Dür, G. Vidal, J.I. Cirac, Phys. Rev. A 62, 062314 (2000)
34. F.G.S.L. Brandao, M.B. Plenio, Nat. Phys. 4, 873 (2008)

35. S. Hill, W.K. Wootters, Phys. Rev. Lett. 78, 5022 (1997)

36. B.V. Fine, F. Mintert, A. Buchleitner, Phys. Rev. B 71, 153105 (2005)

37. S. Alexander, P.W. Anderson, Phys. Rev. 133, A1594 (1964)

38. J. Stöhr, H. Siegmann, Magnetism: from Fundamentals to Nanoscale Dynamics. Springer series in solid-state sciences (Springer, 2006)

39. T. Kostyrko, B.R. Bułka, Phys. Rev. B 84, 035123 (2011)

40. R. Pauncz, The Construction of Spin Eigen-Functions: An Exercise Book (Kluwer Academic/Plenum Publishers, 2000)

41. M. Seo, H.K. Choi, S.Y. Lee, N. Kim, Y. Chung, H.S. Sim, V. Umansky, D. Mahalu, Phys. Rev. Lett. 110, 046803 (2013)

42. N. Linden, S. Popescu, A.J. Short, A. Winter, Phys. Rev. E 79, 061103 (2009)

43. K.M. O'Connor, W.K. Wootters, Phys. Rev. A 63, 052302 (2001)

44. V. Coffman, J. Kundu, W.K. Wootters, Phys. Rev. A 61, $052306(2000)$

45. X. Wang, H. Fu, A.I. Solomon, J. Phys. A: Math. Gen. 34, 11307 (2001)

46. D. Gunlycke, V.M. Kendon, V. Vedral, S. Bose, Phys. Rev. A 64, $042302(2001)$

Open Access This is an open access article distributed under the terms of the Creative Commons Attribution License (http://creativecommons.org/licenses/by/3.0), which permits unrestricted use, distribution, and reproduction in any medium, provided the original work is properly cited. 